\title{
Estado nutricional y ganancia de peso en gestantes
}

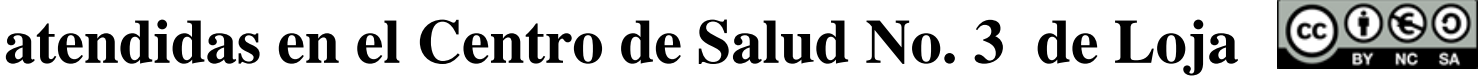

\author{
Nutritional status and weight gain in pregnant women attended at the Loja
} Health Center No. 3

\section{María de los Ángeles Sánchez Tapia. ${ }^{1}$, Cinthya Yazmín Camacas Duarte. ${ }^{2}$ \\ Recibido:01-12-2019 / Revisado: 02-01-2020 /Aceptado: 18-01-2020/ Publicado: 07-02-2020}

\section{Abstract DOI: https://doi.org/10.33262/concienciadigital.v3i1.1.1149}

The initial nutritional status (INS) and the control of weight gain (WG) during the pregnancy are of vital importance, since its alteration constitutes a risk factor for the development of maternal and fetal complications in the short, medium and long term. The objectives were to assess the pre-pregnancy and gestational nutritional status up to the twelfth week of the women assisted at the Health Center No.3 of Loja, and estimate their WG per quarter and establish the relationship of nutritional status and their WG. The study is descriptive. The sample was of 92 pregnant women who met the inclusion criteria. Data was collected from WG curves and Body Mass Index (BMI) of the Ministry of Public Health (MSP), contained in the medical records of pregnant women. After analyzing the information, it was obtained, an initial average $\mathrm{BMI}(\tilde{\mathrm{x}})$ of $25,28 \mathrm{Kg} / \mathrm{m} 2( \pm$ 4.25DE), the population was divided into, $56.5 \%$ with normal INS, $28,3 \%$ overweight, $14,1 \%$ obesity and $1,1 \%$ underweight, the $\mathrm{WG}$ at the end of the third quarter was normal at $44,6 \%$, less than what was recommended at $30,4 \%$ and excessive at $27,2 \%$. It was found that there is no significant difference $(\mathrm{p}>0.05)$ for the relationship between INS and WG at the end of gestation. In conclusion, the initial BMI is not a predictor of the final WG that the pregnant woman will achieve at the end of this process and the alteration of this variable could be determined by other factors.

Keywords: Body Mass Index, Pregnancy, Complication.

\footnotetext{
${ }^{1}$ Doctora en Medicina y Cirugía, Especialista en Ginecología y Obstetricia, Docente de la Facultad de Salud Humana de la Universidad Nacional de Loja, Loja Ecuador marjha123@gmail.com

${ }^{2}$ Médico General, Centro de Salud San Pedro de la Bendita Distrito 11D02 Catamayo Chaguarpamba Olmedo, Loja Ecuador, jazcd@hotmail.com
} 


\section{Resumen}

El estado nutricional inicial (ENI) y el control de la ganancia de peso (GP) durante la gesta, son de vital importancia, ya que su alteración constituye un factor de riesgo para el desarrollo de complicaciones maternas y fetales a corto, mediano y largo plazo. Los objetivos fueron, valorar el estado nutricional pregestacional y gestacional hasta la doceava semana que presentan las mujeres atendidas en el Centro de Salud $N^{\circ} 3$ de Loja, estimar su GP por trimestre y establecer la relación del estado nutricional y su GP. El estudio es descriptivo. La muestra fue de 92 gestantes que cumplieron los criterios de inclusión. Los datos se recogieron de las curvas de GP y de Índice de Masa Corporal (IMC) del Ministerio de Salud Pública (MSP) contenidas en las historias clínicas de las embarazadas. Luego de analizar la información se obtuvo, un IMC inicial promedio( $\left.\tilde{X^{\sim}}\right)$ de $25,28 \mathrm{Kg} / \mathrm{m} 2( \pm 4,25 \mathrm{DE})$, la población se dividió en, 56,5\% con ENI normal, 28,3\% sobrepeso, $14,1 \%$ obesidad y $1,1 \%$ infrapeso; la GP al final del tercer trimestre fue normal en $44,6 \%$, menor a lo recomendado en $30,4 \%$ y excesiva en $27,2 \%$. Se pudo constatar que no existe diferencia significativa $(\mathrm{p}>0,05)$ para la relación entre el ENI y la GP al final de la gestación. En conclusión el IMC inicial no es predictor de la GP final que alcanzará la gestante a término de este proceso y la alteración de esta variable podría estar determinada por otros factores.

Palabras Clave: Índice de Masa Corporal, Embarazo, Complicación.

\section{Introducción}

El estado nutricional de la mujer gestante antes y durante el embarazo constituye un factor determinante para su salud y la del feto, ya sea a lo largo de la gestación, así como en etapas posteriores de la vida. Está determinado por los hábitos maternos, y depende de la satisfacción de las necesidades adicionales, que demanda este proceso fisiológico, así pues se traducirá en un incremento ponderal exitoso, excesivo o deficiente, al final de la gesta, mismo que de estar alterado generará situaciones de riesgo para los dos seres (Organización de las Naciones Unidas para la Agricultura y la Alimentación (FAO), Organización Panamericana de la Salud (OPS), 2017)(Gramage, María, Asins, Álvarez, \& Aguirre, 2013).

Varios estudios a nivel mundial han sabido demostrar que la ganancia ponderal inadecuada durante este proceso, especialmente el exceso, se asocia con la retención de peso materno luego del parto, lo que se perpetúa con la presencia de gestas posteriores y contribuye a la prevalencia del sobrepeso y obesidad entre las mujeres y al riesgo a largo plazo de enfermedades asociadas 
a esta condición (Ronnberg, Ostlund, Fadl, Gottvall, \& Nilsson, 2015), (Haugen et al., 2014), (Cheney, Berkemeier, Sim, Gordon, \& Black, 2017), (Gilmore \& Redman, 2015).

Así alrededor del mundo la nutrición inadecuada constituye uno de los principales problemas de salud y la misma se ha visto asociada al aumento de la mortalidad materna o neonatal desde varias décadas atrás, pues en África Subsahariana y Asia Centro Meridional y Sudoriental, una crecida proporción de mujeres comienza el embarazo con un Índice de Masa Corporal (IMC) superior a $30 \mathrm{Kg} / \mathrm{m}^{2}$, lo que entre otras cosas denota un estado nutricional anormal y acrecienta el riesgo de complicaciones como diabetes gestacional, productos con excesivo o bajo peso al nacer, riesgo aumentado de obesidad infantil e incremento anormal de peso materno, acrecentando aún más el problema de obesidad y sobrepeso en la población general, con todas las comorbilidades que aquello desencadena (Organización Mundial de la Salud (OMS), 2014), (Fouelifack et al., 2015), (Choi, Park, \& Shin, 2011).

En el orden de las ideas anteriores, en Valencia - España, datos de un estudio efectuado a 473 participantes, demostró que el IMC promedio que presentan las gestantes, antes de la semana 12 , corresponde a $24 \mathrm{Kg} / \mathrm{m}^{2}( \pm 5 \mathrm{DE})$. Es así que $12,7 \%$ del total corresponde a bajo peso, $53,7 \%$ normopeso, $23,3 \%$ sobrepeso y $10,4 \%$ obesidad, lo que demuestra un predominio del estado nutricional normal, sin embargo las proporciones de sobrepeso y obesidad son elevadas para lo esperado y en su defecto, el porcentaje de bajo peso es exagerado, considerando los esfuerzos actuales de combatir la malnutrición (Gramage et al., 2013).

En un estudio de cohortes retrospectivo realizado para examinar la tendencia en el aumento de peso durante el embarazo, en Carolina del Norte, encontró que la proporción de mujeres que gana peso gestacional excesivo (más de $18 \mathrm{Kg}$ ) aumentó de 15,5\% a 19,5\% en 5 años. (Muktabhant, Ta, Lumbiganon, \& Laopaiboon, 2015)

Considerando las ideas anteriores es que el reciente informe del Instituto de Medicina (OIM) resume la situación estableciendo que las mujeres de hoy poseen mayor peso; por ende un mayor porcentaje de ellas están iniciando el embarazo con alteraciones en la nutrición, como sobrepeso y obesidad, y resultado de esto es el aumento ponderal exagerado durante la gesta que al final solo conlleva a más problemas de salud. (Muktabhant et al., 2015)

Estas tendencias, en cuanto a aumento ponderal en la gestación, varían de forma significativa, mientras unas investigaciones aseguran que las mujeres obesas tienden a ganar peso en exceso, otros estudios sugieren en cambio que la ganancia de peso es mínima e incluso que existe pérdida ponderal. En una revisión retrospectiva realizada en Estados Unidos, se concluyó que $3,6 \%$ (12 de 328) del total de mujeres con obesidad, de una muestra de 2011 usuarias, perdieron 
peso, en comparación con el grupo de estado nutricional normal $(\mathrm{n}=1227)$, y el de sobrepeso $(\mathrm{n}=456)$ donde el porcentaje de incremento ponderal inadecuado alcanzó solo el $0,08 \%$ y el $0,2 \%$ respectivamente. (Furber et al., 2013)

Por su parte, en Latinoamérica, un estudio transversal realizado en Cartagena Colombia, con una muestra de 413 embarazadas, reportó que el estado nutricional es normal solo en el 42,1\% del total, es decir más de la mitad de gestantes presenta alteraciones en la nutrición. (López Sáleme et al., 2012)

En contraste a aquello, en un estudio realizado en Paraguay, con 75 participantes se reportó que el 68\%(n=51) de gestantes presenta un IMC inicial normal, el 18,7\% ( $n=14)$ obesidad y el $12 \%(n=9)$ bajo peso. Así también se hace referencia al IMC pregestacional promedio, mismo que alcanza valores de $24 \mathrm{Kg} / \mathrm{m}^{2}$ ( \pm 4DE) lo que denota una población en el rango de la normalidad, pero con una ligera tendencia al sobrepeso. (Mendoza, Pérez, \& Sánches, 2010)

En Perú, otra investigación llevada a cabo con 134 gestantes, revela datos diferentes, donde, el 1,5\% ( $n=2)$ de la población estudiada presentó bajo peso, el 49,3\% (n=66) normopeso, el $32,1 \%(n=43)$ sobrepeso y el 17,2\% (n=23) obesidad. De igual manera se hace referencia al IMC pregestacional y hasta el primer trimestre promedio, que en este caso fue de $25,79 \mathrm{Kg} / \mathrm{m}^{2}$ ( \pm 4,8DE) (Magallanes, Barazorda, Quispe, Robles, \& Apaza, 2014)

En el orden de las ideas anteriores, en Ecuador el bajo peso materno ha disminuido en las últimas dos décadas, pero la prevalencia de sobrepeso en este grupo ha tenido un aumento constante, así lo evidencia el Sistema de Vigilancia Alimentaria Nutricional (SISVAN) del Ministerio de Salud Pública (MSP) del Ecuador, mismo que registra un total de 68900 mujeres con detección de su embarazo durante el año 2014, de las cuales el 6,46\% presentaron bajo peso, 51,57\% normopeso, 13,18\% sobrepeso y el 28,78\% obesidad (Ministerio de Salud Pública (MSP), 2014)(Ministerio de Salud Pública (MSP) / Sistema de Vigilancia Alimentaria y Nutricional (SISVAN), 2014a).

Así también, en lo referente a ganancia de peso, reporta un total de 48336 mujeres embarazadas registradas el mismo año, de las cuales el 36,78\% obtuvo una ganancia de peso al final de la gesta adecuado, el 18,87\% excesivo y el 44,35\% deficiente (Ministerio de Salud Pública (MSP) / Sistema de Vigilancia Alimentaria y Nutricional (SISVAN), 2014b).

Continuando con la idea anterior, en la provincia de Loja durante el año 2014 se registra 2 386 mujeres que acuden a consulta para iniciar el control de su embarazo, obteniéndose que de aquellas, 5,07\% presentó bajo peso, 55,53\% normopeso, 9,89\% obesidad y el 29,51\% sobrepeso, así también se registran 2248 gestantes a término, de las cuales el 41,68\%(n=937) 
presentó un aumento ponderal al final de la gesta, catalogado como deficiente, el 41,10\% $(n=924)$ obtuvo una ganancia de peso normal y el 17,22\% $(n=387)$ un aumento de peso excesivo (Ministerio de Salud Pública (MSP) / Sistema de Vigilancia Alimentaria y Nutricional (SISVAN), 2014b) (Ministerio de Salud Pública (MSP) / Sistema de Vigilancia Alimentaria y Nutricional (SISVAN), 2014a).

Por lo expuesto queda claro que la normativa de ganancia ponderal durante el embarazo no ha sido cumplida con éxito en años pasados, constituyéndose un problema de salud para este grupo vulnerable, así mismo se observa que el estado nutricional pregestacional tampoco se ha controlado, hecho preocupante considerando su importancia para la prevención de complicaciones durante y después del embarazo. A nivel local, más del 37\% de los embarazos son no planificados, situación que es común a muchos otros países, por ello es casi imposible realizar una intervención previa sobre la nutrición, y las medidas por ende deben orientarse hacia el aumento ponderal saludable (Soliz, 2013) (Ministerio de Salud Pública (MSP), 2014) (Chávez, Smeke, Rodríguez, Bermúdez, \& Restrepo, 2011), (Krukowski et al., 2016).

En el orden de lo manifestado, es entonces responsabilidad del equipo de salud trabajar junto a la mujer gestante para establecer una buena ganancia ponderal, que se enmarque en las pautas establecidas a nivel nacional y se encamine a preservar en todo momento el bienestar de ambos seres (Tarqui Mamani, Álvarez Dongo, \& Gómez Guizado, 2014).

El MSP ha implementado protocolos para el control de estas variables, y a partir de ello es que la presente investigación se orienta a la evaluación de tales parámetros, que aunque siendo modificables aún hoy, constituyen un serio problema de salud.

Por lo citado y después de efectuar una búsqueda de estudios actuales que evalúen el presente problema de la mujer embarazada, se constató que la información con respecto a esta temática en la localidad es escasa.

En este sentido y tomando en cuenta los esfuerzos que se realizan en el país, con el fin de asegurar el bienestar materno fetal, surgió la siguiente pregunta de investigación, ¿Cómo es el estado nutricional y ganancia de peso durante el embarazo en mujeres que asisten al Centro de Salud $N^{\circ} 3$ de la ciudad de Loja?

Y en base a aquella, se formuló el objetivo general: Determinar el estado nutricional y ganancia de peso en gestantes atendidas en el Centro de Salud $N^{\circ} 3$ de Loja, a través del cumplimiento de los siguientes objetivos específicos: Valorar el estado nutricional pregestacional y gestacional hasta la doceava semana que presentan las mujeres atendidas en el Centro de Salud $\mathrm{N}^{\circ} 3$ de Loja; Estimar la ganancia de peso por trimestre en mujeres gestantes 
atendidas en el Centro de Salud $N^{\circ} 3$ de Loja y Establecer la relación del estado nutricional y la ganancia de peso de la mujer gestante.

\section{Materiales y métodos}

Tipo de estudio. El presente estudio es de tipo descriptivo, transversal con enfoque cuantitativo.

Unidad de estudio. Mujer embarazada

Universo. Conformado por 406 mujeres embarazadas que acudieron al Centro de Salud $\mathrm{N}^{\circ} 3$ de Loja durante el año 2016.

Muestra. El tamaño de muestra fue de 92 mujeres embarazadas que acudieron al Centro de Salud N³ de Loja, durante el período enero - diciembre 2016.

\section{Criterios de inclusión}

- Mujeres que detectaron su embarazo antes de las doce semanas en el Centro de Salud №3.

- Gestantes que realizaron sus controles prenatales en el Centro de Salud N³.

- Gestantes que presentaron embarazo único y con cualquier tipo de paridad.

\section{Criterios De Exclusión}

- Gestantes en cuyas carpetas no conste el registro de datos en la curva de valoración de Índice Masa Corporal pre gestacional y hasta el primer trimestre, así como el peso al final del tercer trimestre.

- Gestantes que después del primer control no hayan vuelto a consulta.

- Gestantes que posean morbilidades previas a la gestación.

\section{Técnicas e instrumentos de recolección de datos}

Se realizó la revisión de historias clínicas de mujeres gestantes cuyo embarazo fue detectado durante el período enero-diciembre 2016 en el Centro de Salud N 3 de la ciudad de Loja.

Para el cumplimiento de todos los objetivos se utilizó como instrumento, la hoja de recolección de datos, elaborada por las autoras en base a directrices previamente establecidas. En esta constan datos de información general como edad en años, edad gestacional al diagnóstico y número de historia clínica, así como el peso, talla e Índice de Masa Corporal (IMC) iniciales, además del peso y la ganancia ponderal por trimestres.

Durante la recolección de información, se registraron los datos generales antes mencionados y luego parámetros como el peso en kilogramos $(\mathrm{Kg})$ y talla en metros $(\mathrm{m})$, a partir de los cuales se calculó el IMC, y así se estableció el estado nutricional inicial, expresado como bajo peso $\left(<18,5 \mathrm{Kg} / \mathrm{m}^{2}\right)$, normal $\left(18,5-24,9 \mathrm{Kg} / \mathrm{m}^{2}\right)$, sobrepeso $\left(25-30 \mathrm{Kg} / \mathrm{m}^{2}\right)$ y obesidad $\left(>30 \mathrm{Kg} / \mathrm{m}^{2}\right)$ 
según lo informa la Organización Mundial de la Salud (OMS) y la Guía de Práctica Clínica del Ministerio de Salud Pública (MSP) del Ecuador.

Luego se procedió a revisar las hojas con las curvas de registro de ganancia de peso del MSP, y se registró la ganancia ponderal total en $\mathrm{Kg}$ por trimestre de gestación. Para propósitos del presente estudio se consideró tres edades, es así que se valoró la ganancia de peso a las 14, 28 y 39 semanas, este último valor considerado como punto medio del intervalo adecuado para el término de la gesta (37 a 42 semanas) ya que en su mayoría y por distintos factores este proceso termina idealmente en este tiempo.

Los datos obtenidos por las mujeres en este periodo fueron comparados con la ganancia ponderal normal para su respectiva categoría según su IMC pre-gestacional y hasta el primer trimestre, tomando como referencia lo establecido en la norma del MSP, considerando como normal lo siguiente:

Tabla 1

Ganancia de peso $(\mathrm{Kg})$ recomendada a la semana14, 28 y 39 del embarazo

\begin{tabular}{cccc}
\hline \multirow{2}{*}{$\begin{array}{c}\text { IMC } \\
\left(\mathbf{K g} / \mathbf{m}^{2}\right)\end{array}$} & \multicolumn{3}{c}{$\begin{array}{c}\text { Ganancia de peso recomendada al } \\
\text { final de cada trimestre }\end{array}$} \\
\cline { 2 - 4 } & $\begin{array}{c}\text { Primero } \\
(\mathbf{K g})\end{array}$ & $\begin{array}{c}\text { Segundo } \\
\text { (Kg) }\end{array}$ & $\begin{array}{c}\text { Tercero } \\
(\mathbf{K g})\end{array}$ \\
Bajo peso & 2,7 a 2,9 & 8 a 11 & 12,1 a 17,4 \\
Normal & 2 a 2,1 & 7,1 a 9,6 & 11,1 a 15,5 \\
\hline Sobrepeso & 1,1 a 1,3 & 4,3 a 6,8 & 6,76 a 11,15 \\
Obesidad & 0,7 a 2,3 & 3,0 a 5,9 & $4,8-8,7$ \\
\hline
\end{tabular}

Fuente: Guía de práctica Clínica del Ministerio de Salud Pública del Ecuador (Ministerio de Salud Pública (MSP), 2014) página 67

Por último se estableció la relación entre el estado nutricional inicial, con la ganancia de peso al final del III trimestre a través de $\mathrm{Chi}^{2} \mathrm{y}$ valor de $\mathrm{p}$.

\section{Resultados}

El promedio de Índice de Masa Corporal (IMC) pregestacional y hasta la doceava semana, encontrado en la presente población durante este período de tiempo fue de $25,28 \mathrm{Kg} / \mathrm{m} 2$ $( \pm 4,25 \mathrm{DE})$.

Así pues de las 92 mujeres que conformaron la muestra el 1,1\% $(\mathrm{n}=1)$ presento bajo peso, $56,5 \%(\mathrm{n}=52)$ presentó peso normal, 23,3\% $(\mathrm{n}=26)$ sobrepeso y $14,1 \%(\mathrm{n}=13)$ obesidad.

En cuanto a la ganancia de peso se refiere, durante el primer trimestre el 16,3\% ( $n=15)$ obtuvieron una ganancia de peso normal, 73,9\% $(\mathrm{n}=68)$ presentó una ganancia ponderal menor a lo recomendado y el 9,8\% (n=9) más de lo esperado. Durante el segundo trimestre 29,3\% 
$(\mathrm{n}=27)$ presento una ganancia ponderal normal, 45,7\% $(\mathrm{n}=42)$ menor a lo recomendado y el $25 \%(\mathrm{n}=23)$ mayor al recomendado, por último y para el tercer trimestre el 38\% $(\mathrm{n}=35)$ presento una ganancia ponderal normal, el 30,4\% $(n=28)$ menor a lo recomendado y el 31,5\% $(n=29)$ más de lo recomendado.

Al referirnos a la estimación de ganancia de peso por grupo nutricional, la media $(\mathrm{X})$ de peso para todos los grupos durante el primer trimestre, se encontró alterada con tendencia a ser menor respeto a las recomendaciones establecidas por el Ministerio de Salud Pública del Ecuador (MSP). Para el segundo trimestre se presentó alterado sólo en el grupo de normopeso, en las gestantes con bajo peso, sobrepeso y obesidad se situó dentro de los rangos recomendados por el MSP. Por último, durante el tercer trimestre aumento el porcentaje de mujeres que obtuvieron una media de peso ideal, sin embargo esta cifra se presentó en menos de la mitad del total.

En el orden de lo mencionado y como punto final de esta investigación, al relacionar el estado nutricional inicial con la ganancia de peso al final del embarazo no se evidencia diferencia significativa entre el grupo de mujeres con estado nutricional normal y aquellas con estado nutricional alterado ya que el valor de " $p$ " es > 0,05; lo que indica que el IMC inicial no es predictor de la ganancia ponderal que alcanzará la mujer gestante al término de este proceso.

\section{Discusión}

$\mathrm{Al}$ estudiar la relación entre el estado nutricional (EN) pregestacional y gestacional hasta la doceava semana, con la ganancia de peso (GP) total al final de la gestación, no se encontró significancia estadística para la correspondencia entre ambas variables, es decir, el EN inicial, no determina el aumento de peso que obtendrá la embarazada durante este proceso.

Como se ha mencionado, el Índice de Masa Corporal (IMC) de la mujer en edad fértil, constituye un factor de vital importancia para el desarrollo y resultado del posible embarazo, luego de constatar la realidad local, es bien sabido que la planificación previa, al momento de procrear no es frecuente y por ende el consejo preconcepcional generalmente no es posible, ocasionando gestas de alto riesgo, con todas las complicaciones que ello conlleva (Ministerio de Salud Pública (MSP), 2012).

En el orden de lo mencionado, el presente trabajo pudo constatar que el IMC pregestacional y gestacional hasta la doceava semana promedio para esta población, fue de $25,28 \mathrm{Kg} / \mathrm{m}^{2}( \pm$ $4,25 \mathrm{DE})$ dato que es similar a lo reportado en Perú, donde corresponde a $25,79 \mathrm{Kg} / \mathrm{m}^{2}( \pm 4,8 \mathrm{DE})$ (Magallanes et al., 2014), pero que se diferencia de los valores obtenidos para países como, África Subsahariana y Asia Centro Meridional y Sudoriental $\left(30 \mathrm{~kg} / \mathrm{m}^{2}\right)$, Valencia España 
$\left(24 \mathrm{Kg} / \mathrm{m}^{2} \pm 5 \mathrm{DE}\right)$ y Paraguay $\left(24 \mathrm{Kg} / \mathrm{m}^{2} \pm 4 \mathrm{DE}\right)$ (Mendoza et al., 2010), (Organización Mundial de la Salud (OMS), 2014), (Gramage et al., 2013).

En ese mismo sentido la población de la presente investigación resultó dividida de la siguiente manera, 56,5\% presentó un EN preconcepcional y hasta la doceava semana normal, el 28,3\% presentó sobrepeso, el 14,1\% obesidad y el 1,1\% bajo peso.

La información obtenida se parece a la de estudios realizados a nivel mundial, así tenemos el efectuado en Valencia España, donde también existió predominio del EN normal (53,7\%), seguido de sobrepeso $(23,3 \%)$ y de obesidad (10,4\%), sin embargo, existió diferencia en las cifras de bajo peso ya que en el presente estudio resulto mínimo, mientras que en la citada población representó el 12,7\%, dato alarmante considerando los esfuerzos actuales de combatir la desnutrición (Gramage et al., 2013).

Por su parte a nivel latinoamericano existen datos que contrastan con los obtenidos en esta investigación, por un mayor porcentaje de mujeres con EN normal, encontramos así que en Paraguay, un estudio realizado con 75 participantes reportó un $68 \%$ de gestantes enmarcadas en la normalidad, $18,7 \%$ con obesidad y $12 \%$ con bajo peso. En Costa Rica, por su parte, se presentó un $64,4 \%$ con normopeso, el $16,7 \%$ con bajo peso, el 7,8\% sobrepeso y el 11,1\% obesidad, lo que indica un predominio de la normalidad aunque las cifras de bajo peso son inadecuadas para lo referido por las Encuestas de Demografía y Salud donde se menciona que el porcentaje para esta condición, no debería ser mayor a 2\% (Organización de las Naciones Unidas para la Agricultura y la Alimentación (FAO), Organización Panamericana de la Salud (OPS), 2017), (Mendoza et al., 2010).

En el orden de lo ya mencionado, los datos obtenidos para Cartagena Colombia, en cambio reportan una proporción menor de normopesas $(42,1 \%)$, de igual manera en Perú, se revela solo el 49,3\% de gestantes enmarcadas en la normalidad, lo que denota claramente que más de la mitad de mujeres gestantes presenta alteraciones en la nutrición, a diferencia de lo obtenido en la presente investigación (López Sáleme et al., 2012).

Al mencionar la localidad cabe destacar las cifras aportadas por el Sistema de Vigilancia Alimentaria Nutricional (SISVAN) del Ministerio de Salud Pública (MSP) del Ecuador para la provincia de Loja durante el año 2014, mismas que registran un total de 2386 mujeres que acuden a consulta para iniciar el control de su embarazo, obteniéndose datos que son similares a los del presente estudio en cuanto a la normalidad (55,53\%), sobrepeso (29,51\%) y obesidad $(9,89 \%)$, pero que contrastan con los reportados para el grupo de bajo peso $(5,07 \%)$ donde la 
proporción es más elevada que la obtenida actualmente (Ministerio de Salud Pública (MSP) / Sistema de Vigilancia Alimentaria y Nutricional (SISVAN), 2014b).

Al hablar de la ganancia ponderal durante la gestación, en nuestro estudio se pudo observar que al final del primer trimestre independientemente del EN, fue en su mayoría deficiente, ya que esta condición representó a más de la mitad de la población estudiada (73,9\%, n=68).

$\mathrm{Al}$ referirse de forma particular a cada grupo, la estimación de GP para el I trimestre en las mujeres de bajo peso fue de $-0,5 \mathrm{Kg}( \pm 0 \mathrm{DE})$, cuando lo recomendado es de 2,7 a $2,9 \mathrm{Kg}$, para las normopesas correspondió a $0,65 \mathrm{Kg}( \pm 2,04 \mathrm{DE})$, cuando lo establecido es de 2 a $2,1 \mathrm{Kg}$, de la misma forma las mujeres con sobrepeso ganaron como media $0,27 \mathrm{Kg}( \pm 1,97 \mathrm{DE})$, cuando lo normal es de 1,1 a $1,3 \mathrm{Kg}$ y por último siguiendo la tendencia anterior las obesas obtuvieron un promedio de ganancia ponderal de $-0,3 \mathrm{Kg}( \pm 3,01 \mathrm{DE})$ cuando su cifra ideal es de 0,7 a 2,3Kg. Como es evidente más de la mitad de mujeres en cada grupo nutricional presenta déficit de peso a esta edad gestacional, mismo que podría ser justificado por el malestar propio de la gesta presente en el primer trimestre, representado por el vómito, así también las náuseas que impiden una buena alimentación y tienden a detener la GP e incluso a ocasionar pérdida ponderal.

Los resultados obtenidos para el segundo trimestre denotan como en el primer momento, un déficit en el aumento ponderal, evidenciado en el 45,7\% $(n=42)$ del total, sin embargo se puede observar que la proporción de mujeres con valores de GP normal aumentaron, pasando de un $16,3 \%(n=15)$ en el I trimestre a 29,3\%(n=27) en el segundo, de igual manera el porcentaje de aquellas con ganancia excesiva de peso incrementó desde 9,8\% (n=9) hacia un 25\% (n=23).

De forma particular este cambio en la proporción se evidenció por la estimación de GP, así pues las mujeres normopesas, obtuvieron una media de $6,9 \mathrm{Kg}( \pm 2,9 \mathrm{DE})$ cuando lo ideal es de 7,1 a $9,6 \mathrm{Kg}$, a diferencia de las usuarias con bajo peso que aumentaron en promedio10 $\mathrm{Kg}( \pm$ ODE), cifra dentro de los rangos establecidos ( 8 a $11 \mathrm{Kg}$ ), las de sobrepeso que obtuvieron $5,3 \mathrm{Kg}( \pm 3,13 \mathrm{DE})$, peso también adecuado $(4,3$ a $6,8 \mathrm{Kg})$ y las obesas cuyo aumento de peso fue en promedio de $4,6 \mathrm{Kg}( \pm 1,99 \mathrm{DE})$ y que de igual manera se situaron dentro de los rangos recomendados $(3$ a $5,9 \mathrm{Kg})$.

Como es evidente, las cifras obtenidas en un primer momento de carácter desalentador, van cambiando mientras transcurre la gesta, dejando atrás la falta ponderal y evolucionando a mejores resultados, debido en buena parte a que, el malestar antes experimentado, para este segundo momento ya ha desaparecido en la mayoría de gestantes.

En el orden de las ideas anteriores, por su parte, los datos obtenidos para el final del tercer trimestre, que para propósitos de este estudio se ha considerado como la semana 39 de 
gestación, arrojaron resultados un tanto desalentadores, ya que se evidencia que menos de la mitad de gestantes, $(44,6 \%, n=41)$, presenta una ganancia ponderal adecuada, el porcentaje restante que en conjunto corresponde a más de la mitad de la población, presenta un aumento de peso anormal, ya sea por exceso, 27,2\% $(n=25)$, o por déficit, 30,4\% $(n=28)$.

Los datos aquí obtenidos se asemejan a lo reportado en países latinoamericanos como Costa Rica, donde se informa que el porcentaje de aumento ponderal adecuado a esta edad gestacional, es solo del 41,7\%, representando menos de la mitad de la población estudiada y los valores de déficit (35\%) y exceso $(23,2 \%)$ en conjunto corresponden a más de la mitad de las gestantes; de igual manera esta realidad se refleja en Perú donde el 40,9\% presenta una ganancia normal, mientras que más de la mitad $(59,1 \%)$ presenta una aumento ponderal inadecuado, lo que se traduce en preocupación y denota un signo de alerta por las comorbilidades a las que este tipo de alteraciones conlleva o puede desencadenar (Apaza, 2015), (Magallanes et al., 2014).

Contrario a lo ya mencionado los resultados del presente estudio y lo citado para países latinoamericanos no concuerda con la realidad a nivel mundial, así tenemos lo reportado para Europa, donde solo del 20 a $40 \%$ de las mujeres gestantes poseen un aumento ponderal anormal, el resto de la población, correspondiente al 60\%, presentan una GP normal; así mismo esta lo encontrado en Carolina del Norte donde la proporción de alteraciones en la GP es muy baja $(19,5 \%)$ (Muktabhant et al., 2015) y lo citado para Aragón España donde se informa que el $55,1 \%$ de la población estudiada obtiene una GP adecuada (Ramon, Abadia, \& Gomez, 2017).

Es preciso mencionar también las cifras reportadas por el SISVAN para la provincia de Loja durante el año 2014, donde se registraron 2248 gestantes a término, de las cuales el 41,68\% presentó un aumento ponderal al final de la gesta catalogado como deficiente, el 41,10\% obtuvo una GP normal y el 17,22\% un aumento de peso excesivo, lo que claramente es similar a lo encontrado en la actualidad, dato a recalcar considerando que aunque esta situación ha sido conocida en años pasados, aún continua ocasionando problema (Ministerio de Salud Pública (MSP) / Sistema de Vigilancia Alimentaria y Nutricional (SISVAN), 2014a).

Continuando las ideas anteriores y de forma específica en cada grupo nutricional, las mujeres que iniciaron con bajo peso, al final del III trimestre, ganaron un promedio de $15 \mathrm{Kg}( \pm 0 \mathrm{DE})$ rango dentro de los límites establecidos por el MSP (12,5 a $18 \mathrm{Kg}$ ), así mismo los demás grupos se encontraron dentro de los rangos establecidos, las normopesas aumentaron $12,2 \mathrm{Kg}( \pm 3,6 \mathrm{DE})$ y lo ideal corresponde de 11,5 a $16 \mathrm{Kg}$, las de sobrepeso $10,3 \mathrm{Kg}( \pm 3,6 \mathrm{DE})$ cuando lo normal es de 7 a $11,5 \mathrm{Kg}$ y las obesas que ganaron un estimado de $8,4 \mathrm{Kg}( \pm 2,7 \mathrm{DE})$ cuando lo óptimo es de 5 a $9 \mathrm{Kg}$. 
Por último y en el orden de desarrollo del presente trabajo, al evaluar la posible relación entre el EN inicial y la ganancia ponderal final se ha constatado que no existe significancia estadística $(\mathrm{p}<0,05)$ para la relación propuesta, es decir, las gestantes del presente estudio, ganaron peso independientemente de su IMC inicial. Esta situación contrasta con algunos estudios realizados a nivel mundial donde se expone una relación entre ambas variables, citando que las mujeres con sobrepeso y obesidad tienden a ganar peso en exceso, mientras que aquellas con bajo peso casi siempre presentan una ganancia ponderal insuficiente (Ortiz Andrellucchi et al., 2014).

Así mismo un estudio realizado en España sobre una muestra de 301 mujeres pudo constatar que el IMC es un factor predictor de la GP gestacional, estableciendo por su parte que las mujeres con sobrepeso u obesidad ganarán menos peso durante la gestación $(\mathrm{p}<0,01)$ y aquellas con bajo peso lo harán en exceso (Ramon et al., 2017), esta asociación es citada también en otro estudio llevado a cabo en Canadá donde se estableció que el IMC previo al embarazo es un predictor significativo del aumento de peso excesivo durante la gesta, datos que de igual manera van en contra de lo obtenido en la presente investigación (Begum, Colman, McCargar, \& Bell, 2012).

A diferencia de lo mencionado, también existen investigaciones como la realizada en Suecia, donde al igual que en el presente estudio, se descarta esta relación, ya que, del total de participantes, solo el $20 \%$ de las embarazadas con obesidad y el $30 \%$ de las mujeres con sobrepeso ganaron peso de forma inadecuada, el resto se enmarcó en la normalidad, de la misma forma se concluye en este misma investigación que el inicio del embarazo con un EN normal no es circunstancia para terminar la gesta en las mismas condiciones, ya que al contrario de lo que se esperaba el 30\% de participantes con IMC adecuado ganaron más de lo que debían, lo que claramente descarta una predisposición obligada a fracasar o tener éxito, según el EN inicial (Muktabhant et al., 2015).

Asi mismo un estudio realizado en Sydney Australia indicó en sus resultados que el riesgo de consecuencias adversas al final de la gesta asociadas a una ganancia ponderal inadecuada es independiente al IMC previo al embarazo (Cheney et al., 2017) (Choi et al., 2011).

En orden de lo mencionado una revisión retrospectiva realizada en Estados Unidos, mostró también la falta de asociación entre ambas variables ya que se constató que el 3,6\% del total de mujeres con obesidad, no sólo no ganó el peso que le correspondía sino que se presentó con pérdida ponderal, lo que ocurrió también para las de EN normal y con sobrepeso en quienes se 
reportó aumento ponderal inadecuado con tendencia al déficit $(0,08 \%$ y el $0,2 \%$ respectivamente) (Furber et al., 2013).

Como puede observarse, el EN inicial de la mujer gestante no influye sobre la ganancia ponderal que la misma terminará obteniendo, por ende el aumento de peso anormal durante este proceso, no constituye una situación inmutable, si no que al contrario puede ser modificada, es allí donde la excelencia de los servicios de salud y una buena atención primaria deben salir a flote, otorgando a la mujer en edad reproductiva y a aquella con una gesta establecida en etapas tempranas, pautas y la educación adecuada con respecto al mantenimiento del proceso fisiológico que constituye el embarazo, dejando claro que el mismo no debe convertirse en una excusa, ni para la indulgencia alimentaria incontrolada, ni para el control excesivo por miedo a perder la figura, sólo así se podrá cambiar el porcentaje de patologías como sobrepeso, obesidad y desnutrición, tanto de la madre como del producto, así como complicaciones que obliguen a realizar procedimientos que de otra forma no se llevarían a cabo, como cesáreas por fetos macrosómicos o que aumentan el riesgo de comorbilidades como diabetes gestacional o preeclampsia.

En ese sentido es también importante entender que el presente problema requiere un abordaje completo e integral, a la mujer gestante. Así mismo se hace hincapié en el valor que tienen los controles prenatales, sobre este grupo, mismos que deberían contar con el tiempo y espacios necesarios, caracterizados por calidad, y que orienten a la madre a desarrollar una gesta de bajo riesgo concientizando en ella y en su familia buenos hábitos de nutrición, lo que contribuirá a disminuir los problemas en este ámbito que en la actualidad se encuentran en auge (Coronel, Nazca, \& Morocho, 2013), (Fealy et al., 2017), (American College of Obstetricians and Gynecologists (ACOG), 2013).

Para terminar, es importante tomar en cuenta, que queda aún mucho por determinar, ya que como se logró demostrar, en esta población la GP inadecuada se presenta en más de la mitad de gestantes, ya sea por exceso o por déficit, esto nos lleva a dejar como interrogante las causas que podrían estar influyendo o determinando las alteraciones en la nutrición de la mujer gestante, como el número y duración de los controles prenatales, escolaridad de la madre, lugar de residencia, factor socioeconómico o creencias culturales. Pues solo así, localizando el origen del problema es que se podrá prevenir, solucionar o al menos disminuir el mismo en el futuro (Deputy, Sharma, Kim, \& Hinkle, 2015). 


\section{Conclusiones}

Más de la mitad de las gestantes presentó un estado nutricional inicial enmarcado en la normalidad, sin embargo, el resto de embarazadas que también representaron un porcentaje considerable se encontraron malnutridas con alteraciones tipo obesidad, sobrepeso y lo que es aún más preocupante bajo peso.

Independientemente del estado nutricional inicial, la ganancia de peso al final del I trimestre fue menor a la recomendada en más de la mitad de gestantes; al finalizar el II trimestre la tendencia hacia la normalidad mejoró, sin embargo la proporción de embarazadas con aumento de peso menor al recomendado continuó representando a la mayoría; por último, al final del III trimestre, el porcentaje de gestantes con incremento ponderal adecuado aumentó, pese a ello, las alteraciones primaron, denotando que más de la mitad de embarazadas no cumplió con la norma de ganancia de peso establecida por el Ministerio de Salud Pública (MSP) al finalizar la gesta.

La asociación del estado nutricional pregestacional y gestacional hasta la doceava semana y la ganancia de peso al final de la gestación, no presentó significancia estadística, es decir, el estado nutricional inicial no es predictor del peso que aumentará la mujer embarazada al final de este proceso.

\section{Agradecimiento}

Al término de esta investigación dejo constancia de mi agradecimiento a la Universidad Nacional de Loja, de manera especial a la carrera de Medicina, en cuyas aulas se llevó a acabo mi formación tanto académica como humanística.

A la Dra. María de los Ángeles Sánchez Tapia, que en calidad de directora de tesis me dirigió con su experiencia profesional, disponibilidad, apoyo y confianza en todo momento y al Dr. Amable Bermeo por su guía para el enfoque del presente trabajo.

A quienes autorizaron los permisos respectivos para la obtención de la información presentada en este trabajo, Dirección Distrital 11D01 Loja Salud y el Centro de Salud N`3 de la ciudad de Loja. 


\section{Referencias Bibliográfica}

American College of Obstetricians and Gynecologists (ACOG). (2013). American College of Obstetrician and Gynecologists. Retrieved May 21, 2019, from https://www.acog.org/Clinical-Guidance-and-Publications/Committee-Opinions/Committeeon-Obstetric-Practice/Weight-Gain-During-Pregnancy ?IsMobileSet=false

Apaza, A. (2015). Ganancia ponderal yanemia en gestantes adolescentes, y estado nutricional del recién nacido 2014. Revista Peruna de Obstetricia Y Enfermeria, 11(1), 7.

Begum, F., Colman, I., McCargar, L. J., \& Bell, R. C. (2012). Gestational Weight Gain and Early Postpartum Weight Retention in a Prospective Cohort of Alberta Women. Journal of Obstetrics and Gynaecology Canada, 34(7), 637-647. http://doi.org/10.1016/S17012163(16)35 316-6

Chávez, N., Smeke, J., Rodríguez, J., Bermúdez, a, \& Restrepo, P. (2011). Estado nutricional en el embarazo y su relación con el peso del recién nacido. Revista Anales Medicos, 56(3), 126 -132 .

Cheney, K., Berkemeier, S., Sim, K. A., Gordon, A., \& Black, K. (2017). Prevalence and predictors of early gestational weight gain associated with obesity risk in a diverse Australian antenatal population: A cross-sectional study. BMC Pregnancy and Childbirth, 17(1), 296. http://doi.org/10.1186/s12884-017-1482-6

Choi, S. K., Park, I. Y., \& Shin, J. chul. (2011). The effects of pre-pregnancy body mass index and gestational weight gain on perinatal outcomes in Korean women: A retrospective cohort study. Reproductive Biology and Endocrinology, 9(1), 6. http://doi.org/10.1186/14777827-9-10

Coronel, E., Nazca, S., \& Morocho, C. (2013). Nutrición de la mujer embarazada y en período de lactancia. MIES, Acción Nutrición, PANI, 10-11. Retrieved from http://www.inclusion.gob.ec/wp-content/uploads/downloads/2013/11/GUIA-1-MADREEMBARAZADA-Y-LACTANCIA.pdf

Deputy, N. P., Sharma, A. J., Kim, S. Y., \& Hinkle, S. N. (2015). Prevalence and Characteristics Associated With Gestational Weight Gain Adequacy. Obstetrics \& Gynecology, 125(4), 773-781. http://doi.org/10.1097/aog.0000000000000739

Fealy, S. M., Taylor, R. M., Foureur, M., Attia, J., Ebert, L., Bisquera, A., \& Hure, A. J. (2017). Weighing as a stand-alone intervention does not reduce excessive gestational weight gain compared to routine antenatal care: A systematic review and meta-analysis of randomised controlled trials. BMC Pregnancy and Childbirth, 17(1), 36. http://doi.org/10.1186/s12884016-1207-2 
Fouelifack, F. Y., Fouedjio, J. H., Fouogue, J. T., Sando, Z., Fouelifa, L. D., \& Mbu, R. E. (2015). Associations of body mass index and gestational weight gain with term pregnancy outcomes in urban Cameroon: a retrospective cohort study in a tertiary hospital. BMC Research Notes, 8(1), 806. http://doi.org/10.1186/s13104-015-1765-9

Furber, C. M., McGowan, L., Bower, P., Kontopantelis, E., Quenby, S., \& Lavender, T. (2013). Antenatal interventions for reducing weight in obese women for improving pregnancy outcome. Cochrane Database of Systematic Reviews, http://doi.org/10.1002/14651858.CD009334.pub2

Gilmore, L. A., \& Redman, L. M. (2015). Weight gain in pregnancy and application of the 2009 IOM guidelines: Toward a uniform approach. Obesity, 23(3), 507-511. http://doi.org/10.1002/oby.20951

Gramage, L., María, B., Asins, A., Álvarez, S., \& Aguirre, A. (2013). Ganancia de peso para un parto sin complicaciones: límite de aumento ponderal según el índice de masa corporal previo. Matronas Profesión, 14(1), 10-16.

Haugen, M., Brantsæter, A. L., Winkvist, A., Lissner, L., Alexander, J., Oftedal, B., ... Meltzer, H. M. (2014). Associations of pre-pregnancy body mass index and gestational weight gain with pregnancy outcome and postpartum weight retention: A prospective observational cohort study. BMC Pregnancy and Childbirth, 14(1), 201. http://doi.org/10.1186/1471-239314-201

Krukowski, R. A., West, D. S., DiCarlo, M., Shankar, K., Cleves, M. A., Saylors, M. E., \& Andres, A. (2016). Are early first trimester weights valid proxies for preconception weight? BMC Pregnancy and Childbirth, 16(1), 357. http://doi.org/10.1186/s12884-016-1159-6

López Sáleme, R., Díaz Montes, C. E., Bravo Aljuriz, L., Londoño Hio, N. P., Salguedo Pájaro, M. D. C., Camargo Marín, C. C., \& Osorio Espitia, E. (2012). Seguridad alimentaria y estado nutricional de las mujeres embarazadas en Cartagena, Colombia. Rev. Salud Pública, 14(2), 200-212. http://doi.org/10.1590/S0124-00642012000200002

Magallanes, M., Barazorda, M., Quispe, J., Robles, R., \& Apaza, A. (2014). Características nutricionales en gestantes del Hospital Nacional Hipólito Unanue, El Agustino 2014. Revista Peruna de Obstetricia Y Enfermeria, (1), 10.

Mendoza, L., Pérez, B., \& Sánches, S. (2010). Estado nutricional de embarazadas en el último mes de gestación y su asociación con las medidas antropométricas de sus recién nacidos. Revista Pediatria Asunción, 37(2), 91-6.

Ministerio de Salud Pública (MSP). (2012). Síntesis de las normas para la prevención de la malnutrición Ecuador 2012. Coordinación Nacional de Nutrición, 122. Retrieved from http://instituciones.msp.gob.ec/images/Documentos/nutricion/Sintesis_Normas.pdf 
Ministerio de Salud Pública (MSP). (2014). Alimentación y nutrición de la mujer gestante y de la madre en período de lactancia. Guía de Práctica Clinica (GPC), 1, 1-80.

Ministerio de Salud Pública (MSP) / Sistema de Vigilancia Alimentaria y Nutricional (SISVAN). (2014a). EVALUACION DEL ÍNDICE DE DIAGNOSTICO PRECONCEPCIONAL 2014.

Ministerio de Salud Pública (MSP) / Sistema de Vigilancia Alimentaria y Nutricional (SISVAN). (2014b). Evaluación del índice de ganancia de peso en embarazadas. Retrieved from http://www.salud.gob.ec/unidad-de-nutricion/

Muktabhant, B., Ta, L., Lumbiganon, P., \& Laopaiboon, M. (2015). Diet or exercise, or both, for preventing excessive weight gain in pregnancy. Cochrane Database of Systematic Reviews, (6), CD007145. http://doi.org/10.1002/14651858.CD007145.pub3.www.cochranelibrary.com

Organización de las Naciones Unidas para la Agricultura y la Alimentación (FAO), Organización Panamericana de la Salud (OPS), O. M. de S. (OMS). (2017). Panorama de la seguridad alimmentaria y nutricional.

Organización Mundial de la Salud (OMS). (2014). Plan de aplicación integral sobre nutrición materna, del lactante y del niño pequeño. Organización Mundial de La Salud, (WHO/NMH/NHD/14.1), 1-30. http://doi.org/10.1007/s13398-014-0173-7.2

Ortiz Andrellucchi, A., Sánchez Villegas, A., Ramírez García, O., Serra Majem, L., Jiménez Acosta, S., Rodríguez Suárez, A., ... Pacheco, J. (2014). Situación nutricional de la gestante y su recién nacido en Cali. An Fac Med., 23(3), 201. http://doi.org/10.1186/1471-2393-14-201

Ramon, E., Abadia, B. M., \& Gomez, S. M. (2017). Ganancia de peso gestacional y retención de peso posparto en una cohorte de mujeres en aragón (España). Nutricion Hospitalaria, 34(5), 1138-1145. http://doi.org/10.20960/nh.749

Ronnberg, A. K., Ostlund, I., Fadl, H., Gottvall, T., \& Nilsson, K. (2015). Intervention during pregnancy to reduce excessive gestational weight gain - A randomised controlled trial. BJOG: An International Journal of Obstetrics and Gynaecology, 122(4), 537-544. http://doi.org/10.1111/1471-0528.13131

Soliz, E. (2013). Ecuador, segundo país en América Latina en embarazo adolescente. Diario El Universo, p. 1. Retrieved from http://www.eluniverso.com/2012/02/24/1/1384/mas-17jovenes-ecuador-son-madres-solo-venezuela-lo-supera-america-latina.html

Tarqui Mamani, C., Álvarez Dongo, D., \& Gómez Guizado, G. (2014). Estado nutricional y ganancia de peso en gestantes peruanas, 2009-2010. An Fac Med., 75(2), 99-105. http://doi.org/10.15381/anales.v75i2.8381 


\section{PARA CITAR EL ARTÍCULO INDEXADO}

Sánchez Tapia, M. de los Ángeles, \& Camacas Duarte, C. Y. (2020). Estado nutricional y ganancia de peso en gestantes atendidas en el Centro de Salud No. 3 de Loja. ConcienciaDigital, 3(1.1), 296-313. https://doi.org/10.33262/concienciadigital.v3i1.1.1149

\section{【L Ciencia}

El artículo que se publica es de exclusiva responsabilidad de los autores y no necesariamente reflejan el pensamiento de la Revista Conciencia Digital.

El artículo queda en propiedad de la revista y, por tanto, su publicación parcial y/o total en otro medio tiene que ser autorizado por el director de la Revista Conciencia Digital.
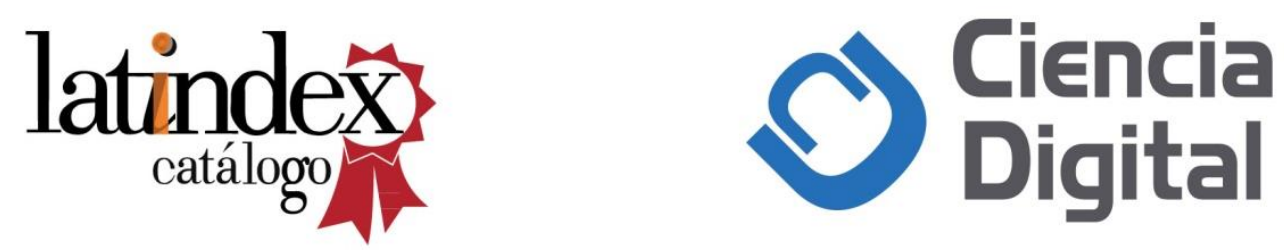